(C) 1983. The Genetical Society of Great Britain

\title{
THE EFFECTS OF HALF-SIB AND MASS SELECTION ON LINKAGE DISEQUILIBRIUM IN RECURRENT SELECTION PROGRAMMES
}

\begin{abstract}
A. J. WRIGHT
Plant Breeding Institute, Maris Lane, Trumpington, Cambridge CB2 2LQ, U.K.

\section{SUMMARY}

The creation of breeding populations of allogamous plants by intermating diverse sources leads to linkage disequilibrium which is an obstacle to improvement by selection. Selection can help to reduce disequilibrium when epistasis favours recombinant over parental gametes, but there is a risk of the loss of useful alleles because of their association with poor gametes. Mass selection has an advantage over half sib selection when dominance is positive because the preferential selection of double and multiple heterozygotes enhances the opportunities for recombination.
\end{abstract}

Received 21.vii.82

\section{INTRODUCTION}

Theoretical work on the expected effects of selection in allogamous species has generally concentrated on populations in linkage (gamete phase) equilibrium or in which any disequilibrium is itself a consequence of earlier selection (Griffing, 1960; Crow and Kimura, 1970). Breeding populations of crop plants are commonly initiated by the intermating of material from a diversity of sources, with the ultimate objective of isolating genotypes in which beneficial alleles from the different sources are combined. In the early generations following such a synthesis, pairs of loci will have arbitrary degrees of disequilibrium, some of which may be very strong and unrelated to the linkage, epistasis or any other genetic properties of the loci concerned. Gardner (1976) has drawn attention to the need for a greater understanding of the interaction of linkage disequilibrium and selection in recurrent selection programmes.

Because it carries the risk of discarding beneficial alleles due to their association with poor gametes, so reducing the genetic potential of the population, selection in these early generations is often avoided. However, logistic and economic pressures may lead to some selection being applied before sufficient generations of random mating have been carried out to restore equilibrium. The purpose of this paper is to point out an important difference between two common methods, mass phenotypic and half-sib selection, in their effects on linkage disequilibrium.

\section{THEORY}

\section{(i) Definitions of gamete values}

During the cycle of random intercrossing following selection, linkage disequilibrium will be diminished by the increase in frequency of recombinant at the expense of parental gametes. Any additional influence due to selection itself will depend on the preferential selection of one or the other type of gamete. Thus, an examination of the process can be carried 
out on the basis of the selective values of the different gametes according to the system of evaluation and selection employed.

Three definitions of gamete values are possible in the parental and offspring generations involved in a single cycle of selection and intercrossing. These are

(a) the weighted mean of the parental zygotes to which the gametic type has contributed $\left(g_{i}\right)$,

(b) the weighted mean of the parental zygotes from which this gamete is regenerated $\left(g_{i}^{\prime}\right)$, and

(c) the weighted mean of the offspring zygotes to which it then contributes, $\left(g_{i}^{\prime \prime}\right)$.

These are therefore

$$
\begin{aligned}
& g_{1}=\sum_{i} f_{j} g_{i}, \\
& g_{i}^{\prime}=\sum_{j} \sum_{k} f_{j} f_{k} g_{i k} t_{(i) j k} / f_{i}^{\prime},
\end{aligned}
$$

and

$$
\mathbf{g}_{i}^{\prime \prime}=\sum_{j} f_{j}^{\prime} g_{i j}
$$

where $f_{i}$ and $f_{i}^{\prime}$ are the frequencies of the $i$ th gametic type in the parental and unselected offspring generations respectively, $g_{i j}$ is the genotypic value of the zygote formed from the $i$ th and $j$ th gametes, and $t_{(i) j k}$ is the frequency of the $i$ th gamete produced from the $j k$ th zygote. Only with linkage equilibrium are these three definitions equivalent.

Considering a pair of loci $(A$ and $B)$ each with two alleles, there are four gametic types, which will be enumerated in the sequence $A B, A b$, $a B, a b$. The coefficient of disequilibrium in the parents is thus $D=$ $f_{1} f_{4}-f_{2} f_{3}$, measuring the excess of coupling gametes over repulsion types, and with a value varying between $-\frac{1}{4}$ and $\frac{1}{4}$. The reasoning used to derive the expectation of $g_{i}^{\prime}$ is essentially the same as that used by Lewontin and Kojima (1960) and later workers to examine the change of gamete frequencies under a model of genotypic fitness. All zygotes other than the double heterozygote produce only the gametic types from which they were formed, so that $t_{(i) j k}=\frac{1}{2}$ when $i=j$ or $k$, and zero otherwise. However, the double heterozygotes reproduce their parental gametes only in the proportion $(1-c)$, where $c$ is the frequency of recombination between the two loci, the remainder being recombinant types. Taking the first gametic type $(A B)$,

$$
\begin{aligned}
g_{1}^{\prime} & =\sum_{j} \sum_{k} f_{j} f_{k} g_{j k} t_{(1) i k} / f_{1}^{\prime} \\
& =\left(f_{1}^{2} g_{11}+f_{1} f_{2} g_{12}+f_{1} f_{3} g_{13}+(1-c) f_{1} f_{4} g_{14}+c f_{2} f_{3} g_{23}\right) / f_{1}^{\prime} \\
& =\left(f_{1} g_{1}-c\left(f_{1} f_{4}-f_{2} f_{3}\right) h\right) / f_{1}^{\prime}=\left(f_{1} g_{1}-c D h\right) / f_{1}^{\prime},
\end{aligned}
$$

where $h$ is the genotype of the double heterozygote $\left(h=g_{14}=g_{23}\right)$. In general,

$$
g_{i}^{\prime}=\left(f_{i} g_{i}-k_{i} c D h\right) / f_{i}^{\prime}
$$

where $k_{i}$ takes a value of 1 for $i=1$ and 4 , and -1 for $i=2$ or 3 . 
The expectation of $g_{i}^{\prime \prime}$ depends on consideration of the changed array of gametes with which it unites at random to produce the offspring zygotes. Because of recombination, the new gamete frequencies are $f_{j}^{\prime}=f_{j}-k_{j} c D$, so that

$$
g_{i}^{\prime \prime}=\sum_{j} f_{i}^{\prime} g_{i j}=\sum_{j}\left(f_{j}-k_{j} c D\right) g_{i j}=g_{i}-c D \sum_{j} k_{j} g_{i j} \text {. }
$$

It should be noted that the mean of the $g_{i}^{\prime}$ is equal to that of the $g_{i}$, since both are defined with respect to the parents, but that

$$
\begin{aligned}
\bar{g}^{\prime \prime} & =\sum_{i} f_{i}^{\prime} g_{i}^{\prime \prime}=\sum_{i}\left(f_{i}-k_{i} c D\right)\left(g_{i}-c D \sum_{j} k_{j} g_{i j}\right) \\
& =\sum_{i} f_{i} g_{i}-2 c D \sum_{i} k_{i} g_{i}+(c D)^{2} \sum_{i} \sum_{j} k_{i} k_{i} g_{i j} \\
& =\bar{g}-c D(2 E-c D L),
\end{aligned}
$$

where

$$
E=\sum_{i} \sum_{j} f_{i} k_{j} g_{i j}=\sum_{j} k_{j} g_{j}
$$

is the epistatic contrast among the gametes which produced the parents and is related to the additive $\times$ additive epistatic effect in that generation, and $L=\sum_{i} \sum_{j} k_{i} k_{j} g_{i j}$ is the dominance $\times$ dominance epistatic genotypic contrast, equal to 41 in the terminology of Mather and Jinks (1971). The change in mean from the parental to offspring generations in the absence of selection is therefore

$$
\bar{g}-\bar{g}^{\prime \prime}=c D(2 E-c D L)
$$

and thus depends on disequilibrium, recombination and epistatis.

In passing, it may be noted that the additional change in population mean brought about by a cycle of phenotypic selection can also be expressed in terms of these gametic effects. Response is a function of the covariance of offspring and parent, which equals the covariance of $g^{\prime}$ and $g^{\prime \prime}$, written as $\sum_{i} f_{i}^{\prime} g_{i}^{\prime} g_{i}^{\prime \prime}-\bar{g}^{\prime} \bar{g}^{\prime \prime}$. Gain can be predicted using the regression of offspring on parent and measured from the mean of the unselected offspring population.

\section{(ii) Selection and disequilibrium}

It has been pointed out that any effect of selection on disequilibrium must depend on the sign of the contrast between coupling and repulsion gametes with respect to their values in the system of selection employed. In the case of mass parental selection, the $g_{i}^{\prime}$ values are appropriate, and their epistatic contrast is

$$
\begin{aligned}
E^{\prime} & =g_{1}^{\prime}-g_{2}^{\prime}-g_{3}^{\prime}+g_{4}^{\prime}=\sum_{i} k_{i} g_{i}^{\prime} \\
& =\sum_{i} k_{i}\left(f_{i} g_{i}-k_{i} c D h\right) / f_{i}^{\prime}=\sum_{i} k_{i} g_{i} f_{i} / f_{i}^{\prime}-c D h\left(\sum_{i} k_{i}^{2} / f_{i}^{\prime}\right) \\
& =\sum_{i} k_{i} g_{i}\left(f_{i}^{\prime}+k_{i} c D\right) / f_{i}^{\prime}-c D h\left(\sum_{i} 1 / f_{i}^{\prime}\right)
\end{aligned}
$$




$$
\begin{aligned}
& =E+c D\left(\sum_{i} g_{i} / f_{i}^{\prime}\right)-c D h\left(\sum_{i} 1 / f_{i}^{\prime}\right) \\
& =E-c D \sum_{i}\left(h-g_{i}\right) / f_{i}^{\prime}=E-c D Q, \text { where } Q=\sum_{i}\left(h-g_{i}\right) / f_{i}^{\prime}
\end{aligned}
$$

This contrast can take a non-zero value even in the absence of epistasis. Kimura (1965) derived an analogous formula relating to a continuous time fitness model and showed that a critical value of $D$ can exist in a natural population when the net effect of recombination and selection is zero. If epistasis and selection are weak, this value represents a stable equilibrium which is maintained over many generations concurrently with changing allele frequencies, and is termed quasi-equilibrium. The present formulation differs in that it deals with recombination only in so far as it is influenced by selection, there being an additional decay of $D$ which continues whether selection is practised or not. When $D$ exceeds $E / c Q$, or is of opposite sign to it, then provided that $Q$ is positive, $E^{\prime}$ will be opposite in sign to $D$ and selection will reinforce recombination in reducing disequilibrium. When $D$ lies between $E / c Q$ and zero, $E^{\prime}$ and $D$ have the same sign and recombination is opposed.

A similar argument can be used to examine the effect of selection based on half-sib means. In this case gametic values are defined with respect to the offspring population, so that

$$
E^{\prime \prime}=\sum_{i} k_{i} g_{i}^{\prime \prime}=E-c D \sum_{i} \sum_{i} k_{i} k_{j} g_{i j}=E-c D L .
$$

The effect of half-sib selection can now be characterised in exactly the same way as that of mass selection, so that it slows the recombination process only when $D$ lies between $E / c L$ and zero. If $L$ is smaller than $Q$, then this region of opposition will be larger for half-sib than for mass selection.

\section{Discussion}

Evidently, either method of selection can have beneficial effects on disequilibrium in a wide range of conditions, but notably for pairs of loosely linked loci whose epistasis $(E)$ and disequilibrium $(D)$ are opposite in sign. Thus the negative disequilibrium of pairs of loci whose beneficial alleles originated from different source populations will be reduced when their interaction is multiplicative or complementary. A prediction as to the effects of selection can be made by comparing the means of parental and offspring generations. If the offspring mean is the higher, then the balance of $c D(2 E-c D L)$ terms over all loci pairs is negative and $D$ and $\left(E-\frac{1}{2} c D L\right)$ are of opposite sign. In most circumstances it can be expected that $(E-c D L)$ and perhaps $(E-c D Q)$ will similarly be of opposite sign to $D$, so that selection of either type will reduce disequilibrium on average over all pairs of loci. If the parental mean is the higher, on the other hand, then selection will tend to oppose recombination and increase $D$.

A rigorous comparison of the two methods of selection would have to take account of the difference in selection pressure each imposes on the various locus pairs, according to their heritabilities and other factors. The present comparison ignores these differences and therefore assumes that 
an equivalent selection is applied to each two locus gametic array by each method. The method with the characteristic of reducing $D$ most consistently and strongly over all conditions is then the one whose gametic epistatic contrast tends to be the larger when opposite in sign to $D$ and the smaller when of the same sign. Because the term involving $D$ is a decrement to both $E^{\prime}$ and $E^{\prime \prime}$, phenotypic selection will be the preferred method when $Q$ exceeds $L$ (i.e., is more positive) and vice versa. This argument is valid whatever the sign and magnitude of $E$.

There are thus two separate mechanisms by which selection influences linkage disequilibrium. The first is the preferential selection of either coupling or repulsion gametes because of gametic epistasis (see also Felsenstein, 1965), and the second is the enhancement or retardation of recombination by the preferential selection or rejection of the double heterozygote. The dominance which is a major part of the phenotypic value of the double heterozygote is suppressed in half-sib family means so that its selective value will tend to lie much closer to the population mean. For pairs of dominant loci, mass selection will tend to reduce any initial disequilibrium and maintain it throughout the programme at a lower level than will half-sib family selection or progeny testing. For pairs of recessive loci, on the other hand, mass selection will be more liable to maintain high levels of disequilibrium, particularly if any initial disequilibrium is of the same sign as the epistatic effect $(E)$. Similar arguments to the above could be advanced with respect to the effect of multiple heterozygotes on the linkage disequilibria among three or more loci.

In practice a number of factors, logistical as well as genetical, will influence the choice of selection method, as in the use of a half-sib system (modified ear-to-row) in the early generations of the maize programme at CIMMYT (Anon, 1975). However, since the majority of important production traits in most crops are controlled by dominant genes, mass selection is expected to be the most effective method for minimising linkage disequilibrium.

\section{REFERENCES}

ANON, 1975. CIMMYT review.

CROW, J. F. AND KIMURA, M. 1970. An Introduction to Population Genetics Theory. Harper and Row Publishers, New York, Evanston and London.

FELSENSTEIN, J. 1965. The effect of linkage on directional selection. Genetics, 52, 349-363.

GARDNER, C. O. 1976. Quantitative genetic research in plants: Past accomplishments and research needs. Proc. Int. Conf. Quant. Genet., Iowa State.

KIMURA, M. 1965. Attainment of quasi linkage equilibrium when gene frequences are changing by natural selection. Genetics, 52, 875-890.

LEWONTIN, R. C. AND KOJIMA, K. 1960. The evolutionary dynamics of complex polymorphisms. Evolution, 14, 458-472. 